\title{
Proposta de um método para estratificação de usuários adscritos por equipe de saúde na Atenção Primária à Saúde: relato de experiência
}

\author{
Proposal of a method for stratification of users enrolled by health team in Primary Health \\ Care: experience report
}

Propuesta de un método de estratificación de los usuarios inscritos por el equipo de salud en Atención Primaria de Salud: informe de experiencia

\begin{abstract}
Elisabet Pereira Lelo Nascimento ${ }^{1 *}$, Silvia Aparecida Maria Lutaif Dolci Carmona1, Vânia Maria Corrêa Barthmann ${ }^{1}$, Júlio César de Moraes .
\end{abstract}

\section{RESUMO}

Objetivo: Relatar a experiência de elaboração de um método de estratificação dos usuários adscritos por equipe de saúde, a partir de um indicador social de acesso público na perspectiva de contemplar a heterogeneidade do território, as desigualdades sociais e o diagnóstico das vulnerabilidades, amparando a primeira questão norteadora da metodologia do dimensionamento da força de trabalho. Relato da experiência: Estudo descritivo do tipo relato de experiência, concebido para superar as dificuldades do dimensionamento em uma região de saúde do Ceará, descrito em quatro etapas para classificar as vulnerabilidades dos territórios de abrangência de cada Unidade de Atenção Primária em Saúde (UAPS): identificação do índice do indicador bolsa família, realização do cálculo da média aritmética simples entre os índices do indicador designado, classificação e definição de vulnerabilidade para cada UAPS e adscrição de usuários para cada grau de vulnerabilidade, variando de 2.000 a 3.500 por equipe de Saúde da Família (eSF). Considerações finais: $O$ método proposto oferece uma valiosa contribuição ao planejamento em saúde, vistas à produção de informações essenciais para subsidiar a formulação de políticas públicas, considerando as diferenças das condições de vida e de saúde da população, e fortalecendo a Estratégia de Saúde da Família (ESF).

Palavras-chave: Atenção Primária à Saúde, Vulnerabilidade social, Estratificação social, Estratégia de Saúde da Família.

\begin{abstract}
Objective: To report the experience of developing a method of stratification of users enrolled by a health team, from a social indicator of public access in order to contemplate the heterogeneity of the territory, social inequalities and the diagnosis of vulnerabilities, supporting the first fundamental question of the methodology of workforce dimensioning. Experience report: Descriptive study of the type of experience report, designed to overcome the difficulties of dimensioning in a health region of Ceará, described in four stages to classify the vulnerabilities of the territories covered by each Primary Health Care Unit (UAPS): identification of the index of the family stock index, calculation of the simple arithmetic mean among the indexes of the designated indicator, classification and definition of vulnerability for each UAPS and adscrition of users for each degree of vulnerability, ranging from 2.000 to 3.500 by family health team (eSF). Final considerations: The proposed method offers a valuable contribution to health planning, with a view to the production of essential information to support the formulation of public policies, considering the differences in living and health conditions of the population, and strengthening the Family Health Strategy (ESF).
\end{abstract}

Keywords: Primary Health Care, Social vulnerability, Social stratification, Family Health Strategy.

1 Universidade Federal de Goiás (UFG), Goiânia - GO. *E-mail: betlelo@uol.com.br

PUBLICADO EM: 2/2021 


\section{RESUMEN}

Objetivo: Reportarla experiencia de desarrollo de un método de estratificación de usuarios inscritos por equipo de salud, desde un indicador social de acceso público para contemplar la heterogeneidad del territorio, las desigualdades sociales y el diagnóstico de vulnerabilidades, apoyando la primera cuestión fundamental de la metodología de dimensionamiento del personal. Informe de experiência: Estudio descriptivo del tipo de informe de experiencia, diseñado para superar las dificultades de dimensionamiento en una región de salud de Ceará, descrito en cuatro etapas para clasificar las vulnerabilidades de los territorios cubiertos por cada Unidad de Atención Primaria de Salud (UAPS): identificación del índice del indicador de subvención familiar, cálculo de la media aritmética simple entre los índices del indicador designado, clasificación y definición de vulnerabilidad para cada UAPS y adscrición de usuarios para cada grado de vulnerabilidad, que oscila entre 2.000 y 3.500 por equipo de salud familiar (eSF). Consideraciones finales: El método propuesto ofrece una valiosa contribución a la planificación de la salud, con miras a la producción de información esencial para apoyar la formulación de políticas públicas, teniendo en cuenta las diferencias en las condiciones de vida y salud de la población, y fortaleciendo la Estrategia de Salud Familiar (FSE).

Palabras clave: Atención Primaria de Salud, Vulnerabilidad social, Estratificación social, Estrategia de Salud Familiar.

\section{INTRODUÇÃO}

O Sistema Único de Saúde (SUS) tem como estratégia a organização descentralizada e capilarizada nas ações e nos serviços de saúde. Compreende a Atenção Primária à Saúde (APS) como uma forma singular de ordenar a rede de atenção à saúde e coordenar a gestão do cuidado centrado no usuário, sendo a porta de entrada preferencial do SUS se responsabilizando pelas ações individuais e coletivas a partir dos arranjos organizacionais dos processos de trabalho das equipes de saúde. Nesse contexto, a Estratégia de Saúde da Família (ESF) tem favorecido a universalização do cuidado, agregando elementos fundamentais para uma APS mais abrangente, como a valorização do acesso, da equidade e da integralidade da atenção (ARANTES LJ, et al., 2016; OLIVEIRA LCC e REIS AAC, 2018).

Dada a relevância e abrangência desse nível de atenção, conjugada com a escassez de literaturas sobre dimensionamento da força de trabalho para a APS que considerem as singularidades de cada território desenvolveu-se uma metodologia ancorada em quatro questões norteadoras: "Para quem?" - para conhecer os usuários nos territórios onde vivem e suas necessidades de saúde; "O que oferecer?" - para identificar os serviços instalados que atendam às necessidades de saúde dos usuários; "Como?"- para identificar o processo organizativo dos serviços, onde são desenvolvidas as atividades de promoção, prevenção e recuperação da saúde; por fim, "Quantos?" - que identifica os profissionais necessários para prestar assistência qualificada e resolutiva aos usuários (VIANNA CMM, et al., 2013; MACHADO CR e DAL POZ MR, 2015, MARQUES AMP, 2016; NASCIMENTO EPL e CARMONA SAMLD,2020).

Esse dispositivo pressupõe um processo de análise dos dados do planejamento local, série histórica e potencial de produção, caracterização da força de trabalho em exercício, distribuição do uso do tempo dos profissionais por processo de trabalho e a capacidade física instalada. O desenvolvimento desta metodologia acontece por meio de uma construção coletiva em conjunto com gestores e trabalhadores das secretarias de saúde, resultando na elaboração do dimensionamento da força de trabalho, como um método contínuo de planejamento (NASCIMENTO EPL, et al., 2020).

Essa ferramenta foi reconhecida pelo Ministério da Saúde, por intermédio do INOVASUS de 2013, e posteriormente desenvolvida em 14 municípios de diversas regiões do País, incorporando novas perspectivas e inovações tecnológicas (NASCIMENTO EPL, et al., 2015). Nesse percurso, verificaram-se vários avanços na área da gestão do trabalho, dentre eles, avaliação da produtividade dos profissionais e da capacidade física instalada, identificação de necessidade de realocação de trabalhadores, conhecimento da força de trabalho existente e necessária, definição de vagas para concursos e reorganização do processo de trabalho (MONCLAR CN, et al., 2020). 
No entanto, alguns entraves relacionados à utilização de indicadores para circunscrever a população usuária da APS, acabaram determinando a escolha de parâmetros pela disponibilidade de obtenção das informações, e não pela relevância deles na caracterização das necessidades de saúde da população e do território (IBICT, 2020; NASCIMENTO EPL, et al., 2020).

À vista disso, o objetivo deste estudo é apresentar a proposta de um método de estratificação dos usuários adscritos por equipe de saúde na Atenção Primária à Saúde, a partir de um indicador social de acesso público na perspectiva de contemplar a heterogeneidade do território, as desigualdades sociais e o diagnóstico das vulnerabilidades, amparando a primeira questão norteadora da metodologia do dimensionamento da força de trabalho.

\section{RELATO DE EXPERIÊNCIA}

O Ministério da Saúde por intermédio do Departamento de Gestão do Trabalho em Saúde, em parceria com o Instituto Brasileiro de Informação em Ciência e Tecnologia (IBICT), desenvolveu, em quatro municípios pertencentes à primeira região de saúde do Ceará, no período de junho de 2019 a fevereiro de 2020, o projetopiloto denominado Governança da Gestão do Trabalho em Saúde, com a finalidade de implementar a metodologia do dimensionamento da força de trabalho em saúde para a rede de atenção à saúde daquela região.

Durante o desenvolvimento do projeto, evidenciou-se um grande desafio resultante da inexistência do mapa de abrangência desse território por Unidades de Atenção Primária à Saúde (UAPS), revelando uma fragilidade ao responder a primeira questão norteadora (para quem?) da metodologia do dimensionamento da força de trabalho para a APS. Os indicadores sociais e epidemiológicos e a adscrição de usuários eram referenciados por bairros, município e região intramunicipal, dificultando a identificação de índices referentes à especificidade de cada território e o diagnóstico das vulnerabilidades existentes, culminando na impossibilidade de estratificar os usuários adscritos por equipe de saúde.

Os obstáculos evidenciados impulsionaram os autores a buscarem alternativas que pudessem solucionar a dificuldade constatada nessa primeira fase do processo do dimensionamento da força de trabalho em saúde da APS. Assim, originou-se a proposta de um método para estratificação de usuários adscritos por equipe de saúde na APS, através de média aritmética simples do indicador social do programa bolsa família, viabilizando uma maior aproximação às singularidades das UAPS do município.

Destarte, a seguir apresentamos a descrição esquemática da estratificação de usuários adscritos por equipe de saúde na APS, a partir do indicador social do programa bolsa família, na perspectiva de contemplar a heterogeneidade do território, as necessidades dos usuários em situação de vulnerabilidade e as desigualdades sociais. Para demonstrar a aplicabilidade do método proposto, adotamos um cenário fictício com unidades da APS, contemplando as informações necessárias ao desenvolvimento desse dispositivo.

A proposta foi descrita em quatro etapas para classificar as vulnerabilidades dos territórios de abrangência de cada UAPS: identificação do índice do indicador bolsa família; realização do cálculo da média aritmética simples entre os índices do indicador designado; classificação e definição de vulnerabilidade para cada UAPS; e adscrição de usuários para cada grau de vulnerabilidade, variando de 2.000 a 3.500 por equipe de Saúde da Família.

A primeira etapa consistiu em identificar o índice do indicador bolsa família de cada UAPS mediante a realização de pesquisa na plataforma e-Gestor Atenção Básica, onde estão disponíveis as informações dos beneficiários acompanhados pelos estabelecimentos de saúde. Posteriormente, na segunda etapa, utilizamos a média aritmética simples, que é usada em estatística como uma medida de tendência central, sendo obtida ao se somar todos os valores e dividindo-se o valor encontrado pelo número de dados desse conjunto por atender o objetivo da estratificação das unidades de saúde (Tabela 1). 
Tabela 1 - Identificação da população total e do índice do indicador bolsa família por UAPS e a média aritmética simples entre os indicadores designados.

\begin{tabular}{ccc}
\hline Unidade de Atenção Primária em Saúde & População Total & Indicador de Bolsa Família \% \\
\hline FORMOSA & 8.745 & 10,3 \\
SANTANA & 7.908 & 13,4 \\
PARAÍSO & 9.187 & 17,5 \\
SÃO VICENTE & 12.354 & 17,9 \\
CAMPO VERDE & 16.892 & 15,2 \\
SANTA LÚCIA & 10.946 & 18,2 \\
AMERICANO & 8.978 & 18,0 \\
SÃO JOAQUIM & 12.585 & 21,9 \\
ALVORADA & 7.569 & 9,5 \\
SANTO ANTÔNIO & 10.104 & 14,1 \\
SANTA CLARA & 14.757 & 10,6 \\
VILA UNIÃO & 10.542 & 13,8 \\
SUÍÇO & 9.143 & 11,2 \\
SÃO BENEDITO & 10.677 & 21,2 \\
\hline Total Geral (14 Unidades) & $\mathbf{1 5 0 . 3 8 7}$ & $\mathbf{2 1 2 , 8}$ \\
\hline Média do Indicador Bolsa Família & - & $\mathbf{1 5 , 2}$ \\
\hline
\end{tabular}

Fonte: Nascimento EPL, et al., 2020.

A terceira etapa compreendeu estabelecer em quantos níveis seriam classificadas as UAPS, atribuindo, posteriormente, os graus de vulnerabilidade e, por fim, definido a adscrição de usuários, utilizando quatro níveis entre os indicadores identificados (Quadro 1).

Quadro 1 - Níveis de classificação das UAPS conforme a vulnerabilidade e adscrição de usuários por eSF sugerida.

\begin{tabular}{|c|c|}
\hline Grau de Vulnerabilidade & Proposta de Adscrição de Usuários por eSF \\
\hline Vulnerabilidade Baixa & 3.500 \\
\hline Vulnerabilidade Média & 3.000 \\
\hline Vulnerabilidade Alta & 2.500 \\
\hline Vulnerabilidade Muito Alta & 2.000 \\
\hline
\end{tabular}

Fonte: Nascimento EPL, et al., 2020.

Para classificar as UAPS, foi necessário encontrar o intervalo entre os indicadores, utilizando a seguinte fórmula:

$$
\text { Intervalo }=\frac{\text { média do indicador bolsa família }}{\text { número de níveis }}
$$

A partir desse resultado, identificou-se o menor índice entre os indicadores, aqui demonstrado pela UAPS Alvorada, e acrescentou-se o valor do intervalo encontrado, definindo o primeiro escore. As outras classificações ocorreram, sucessivamente, a partir do valor subsequente identificado (Quadro 2).

Escore 1 = até [menor índice + intervalo];

Escore 2 = de [escore $1+0,1]$ até [resultado + o intervalo];

Escore $3=$ de [escore $2+0,1]$ até [resultado +0 intervalo];

Escore $4=$ acima de [escore $3+0,1$ ] 
Quadro 2 - Estratificação dos índices do indicador bolsa família por grau de vulnerabilidade e a proposta de adscrição de usuário por equipe da UAPS.

\begin{tabular}{|c|c|c|c|c|}
\hline Cálculo \% & Resultado \% & Escores \% & Vulnerabilidade & $\begin{array}{c}\text { Proposta de Adscrição de } \\
\text { Usuários }\end{array}$ \\
\hline $9,5+3,8$ & 13,3 & Até 13,3 & BAIXA & 3.500 \\
\hline $13,4+3,8$ & 17,2 & De 13,4 a 17,2 & MÉDIA & 3.000 \\
\hline $17,3+3,8$ & 21,1 & De 17,3 a 21,1 & ALTA & 2.500 \\
\hline & & Acima de 21,2 & MUITO ALTA & 2.000 \\
\hline
\end{tabular}

Fonte: Nascimento EPL, et al., 2020.

A partir desse cálculo, procedeu-se a quarta etapa, apresentando o resultado da adscrição de usuários de acordo à classificação das vulnerabilidades, por meio do indicador bolsa família, com estimativa do número de equipes para cada UAPS (Tabela 2).

Tabela 2 - Classificação de vulnerabilidade, adscrição de usuários e dimensionamento de equipes de saúde por UAPS.

\begin{tabular}{cccccc}
$\begin{array}{c}\text { Unidade de Atenção } \\
\text { Primária em Saúde }\end{array}$ & $\begin{array}{c}\text { População } \\
\text { Total }\end{array}$ & $\begin{array}{c}\text { Indicador } \\
\text { Bolsa } \\
\text { Família \% }\end{array}$ & $\begin{array}{c}\text { Classificação } \\
\text { das } \\
\text { Vulnerabilidades }\end{array}$ & $\begin{array}{c}\text { Proposta de } \\
\text { Adscrição de } \\
\text { Usuários }\end{array}$ & $\begin{array}{c}\text { No de } \\
\text { Equipes }\end{array}$ \\
\hline ALVORADA & 7.569 & 9,5 & BAIXA & 3.500 & 2,2 \\
SUÍÇO & 9.143 & 9,9 & BAIXA & 3.500 & 2,6 \\
FORMOSA & 8.745 & 10,3 & BAIXA & 3.500 & 3,5 \\
SANTA CLARA & 14.757 & 10,6 & BAIXA & 3.500 & 4,2 \\
SANTANA & 7.908 & 13,4 & MÉDIA & 3.000 & 2,6 \\
VILA UNIÃO & 10.542 & 13,8 & MÉDIA & 3.000 & 3,5 \\
SANTO ANTÔNIO & 10.104 & 14,1 & MÉDIA & 3.000 & 3,4 \\
CAMPO VERDE & 16.892 & 15,2 & MÉDIA & 3.000 & 5,6 \\
PARAÍSO & 9.187 & 17,5 & ALTA & 2.500 & 3,7 \\
SÃO VICENTE & 12.354 & 17,9 & ALTA & 2.500 & 4,9 \\
AMERICANO & 8.978 & 18,0 & ALTA & 2.500 & 3,6 \\
SANTA LÚCIA & 10.946 & 18,2 & ALTA & 2.500 & 4,4 \\
SÃO BENEDITO & 10.677 & 21,2 & MUITO ALTA & 2.000 & 5,3 \\
SÃO JOAQUIM & 12.585 & 21,9 & MUITO ALTA & 2.000 & 6,3 \\
\hline Total & $\mathbf{1 5 0 . 3 8 7}$ & $\mathbf{2 1 2 , 8}$ & - & - & $\mathbf{5 5 , 8}$ \\
\hline
\end{tabular}

Fonte: Nascimento EPL, et al., 2020.

A adscrição de usuários apresentada foi orientada pelo intervalo sugerido pela Política Nacional da Atenção Básica (PNAB), todavia, pode ser definido outro parâmetro populacional de responsabilidade das equipes de Saúde da Família, de acordo às especificidades dos territórios. Reiteramos a relevância de se considerar os contrastes urbanos e as desigualdades sociais presentes nas áreas de abrangência das UAPS de cada município brasileiro, garantindo a assistência mais qualificada aos usuários. 


\section{DISCUSSÃO}

O método para estratificação de usuários adscritos por equipe de saúde na Atenção Primária à Saúde foi amparado na primeira questão norteadora: "Para quem?" e consideramos o usuário como protagonista, um sujeito que carrega uma história, é ativo em seus processos e habita um determinado lugar com todas as suas convicções e contradições, denominado território vivo, com a sua dinâmica social e os dados naturais modificados pelo homem (SANTOS M, 2000; SANTOS M, 2004; FARIA RM, 2013).

Esse usuário traz um conjunto de necessidades que devem ser consideradas na gestão em saúde, portanto, reconhecer, planejar e fazer a gestão do território é fundamental para a implementação das políticas públicas e para garantir sua eficácia (BEZERRA ACV, 2015). Assim, a PNAB aponta como diretriz a territorialização e adscrição, favorecendo a descentralização e o planejamento das ações de saúde voltadas para as singularidades dos territórios. Ainda, recomenda como forma de garantir a coordenação do cuidado, ampliação de acesso e resolutividade que:

"A população adscrita por equipe de Atenção Básica (eAB) e de Saúde da Família (eSF) seja de 2.000 a 3.500 pessoas, localizada dentro do seu território, garantindo os princípios e diretrizes da Atenção Básica. Além dessa faixa populacional, podem existir outros arranjos de adstrição, conforme vulnerabilidades, riscos e dinâmica comunitária(...)" (BRASIL, 2017).

Entretanto, tal portaria não apresenta orientação ou dispositivo que seja capaz de auxiliar os trabalhadores e gestores municipais na realização da adscrição de usuários, que seja baseada nas vulnerabilidades presentes nos territórios de abrangência das UAPS, atendendo as diretrizes de fortalecimento e consolidação da ESF.

Ressaltamos que, para certificar a validade e potência do indicador bolsa família, foram realizadas algumas análises comparativas entre outros parâmetros e indicadores, obtidos por meio de dados secundários e dados primários, coletados em campo, no período do desenvolvimento do projeto de Governança da Gestão do Trabalho em Saúde, tais como, incidência de tuberculose, taxa de mortalidade infantil e mortalidade por causas externas. Nesses ensaios, o Índice de Desenvolvimento Humano (IDH) confirmou, fortemente, o mosaico de contrastes urbanos e de desigualdades sociais do município (CARVALHO DS e NASCIMENTO EPL, 2020).

Embora o indicador da bolsa família possa não representara totalidade da população vulnerável que deveria estar vinculada ao programa, ele é potente por considerar vulnerabilidades sociais e ser de acesso público, possibilitando a seleção desse indicador para a realização da estratificação de usuários adscritos, por UAPS, de qualquer localidade do Brasil.

Assim, o método proposto para estratificação de usuários adscritos por equipe de saúde considerou as singularidades mencionadas, por intermédio do indicador referente à população vinculada ao programa bolsa família, como uma expressão das desigualdades sociais de um determinado território. Esse indicador sinaliza o cenário do desemprego, da pobreza extrema, da falta de investimento em moradia, de alimentação, de educação, de acesso à saúde, dentre outras necessidades (BRASIL, 2020).

Compreender a vulnerabilidade requer identificar as fragilidades presentes no território, então, o programa bolsa família, instituído pela Lei $\mathrm{n}$. 10.836, de nove de janeiro de 2004, que garante benefícios básicos destinados às famílias em situação de extrema pobreza, institui-se como um indicador socioeconômico mensurável (BRASIL, 2004). Desse modo, possui relevância por se tratar de um programa de transferência de renda e, ainda, por estabelecer o exercício de direitos sociais básicos na área da saúde, ao propor que famílias beneficiadas sejam acompanhadas pelo setor saúde, em especial no âmbito da APS (CARVALHO AT, et al., 2014).

Corroborando a relevância da escolha de um indicador social para o desenvolvimento do método de estratificação, o documento que mostra a análise das condições de vida da população brasileira, a partir de dados da Pesquisa Nacional por Amostra de Domicílios (PNAD), traz a estratificação dos quintos de rendimento em uma de suas análises, apontando que, enquanto apenas $35,7 \%$ da população com maiores 
rendimentos não possuem plano suplementar de assistência médica ou odontológica de saúde, 94,4\% da população com rendimentos menores estão nessa condição (BRASIL, 2016). Com isso, podemos identificar que a desigualdade social em saúde pode ser observada em estratos sociais, com diferentes condições e com índices de saúde distintos, que podem chegar até à iniquidade no acesso aos serviços de saúde.

O sistema de gestão do programa bolsa família concentra os registros das condicionalidades de saúde dos beneficiários acompanhados pelas equipes de saúde, educação e assistência social, por agrupamento de bairro e por estabelecimento de atenção à saúde. $O$ acesso às informações referentes ao programa está disponível na plataforma e-Gestor Atenção Básica, por meio do endereço eletrônico: https://egestorab.saude.gov.br (BRASIL, 2004). Nesse sentido, o indicador apresenta concretude e aproximação à realidade dos usuários adscritos em cada UAPS, é de fácil acesso e, ainda, possibilita consulta de relatórios com os resultados de acompanhamento, utilizados como instrumentos de diagnóstico, avaliação e monitoramento para a atuação do poder público (BRASIL, 2020).

Partindo desse pressuposto que reconhece a dinâmica epidemiológica e social da área de abrangência de uma UAPS, que é o primeiro passo ao planejamento referente à análise situacional do território, o programa bolsa família foi escolhido, como um indicador que representa uma importante estratégia referente às políticas sociais no Brasil.

A estratificação, por sua vez, é uma ferramenta robusta no que tange separar os dados levantados em grupos distintos e tem sido utilizada em estudos na área da saúde, evidenciando adequação ao objetivo das pesquisas. Um estudo recente sobre a estratificação dos municípios brasileiros, em grupos homogêneos, para estudos de avaliação de desempenho da gestão em saúde, a partir de indicadores sociais e de saúde, assegurou coerência entre os diferentes portes populacionais e a classificação por influentes da gestão, agregando valor à proposta de estratificação (CALVO MCM, et al., 2016). A relação entre indicadores de saúde e a estratificação do espaço urbano pelas condições de vida permitem pensar em políticas que ampliem o acesso das populações mais vulneráveis a esses serviços (GOMES DR, et al., 2016).

Esperamos que este trabalho contribua com os gestores e trabalhadores, permitindo a reorganização dos mapas territoriais de abrangência das Unidades de Atenção Primária em Saúde, melhorando o acesso e o cuidado na APS. Recomendamos que, para a utilização deste método, sejam realizados os ajustes necessários e/ou incorporação de outros indicadores e parâmetros, de forma a atender as diretrizes das secretarias municipais de saúde de todo território nacional.

O método proposto ampara a primeira questão norteadora do dimensionamento da força de trabalho da Atenção Primária em Saúde e oferece uma valiosa contribuição ao planejamento em saúde, tendo em vista a produção de informações essenciais que subsidiam a formulação de políticas públicas. Ademais, considera as diferenças das condições de vida e de saúde da população a partir do indicador bolsa família, por meio da estratificação dos usuários adscritos por equipe de saúde na APS. Este dispositivo ainda permite dimensionar as equipes de Saúde da Família, viabilizando uma distribuição mais equânime da força de trabalho e fortalecendo a Estratégia de Saúde da Família, modelo prioritário no País.

\section{REFERÊNCIAS}

1. ARANTES LJ, et al. Contribuições e desafios da Estratégia Saúde da Família na Atenção Primária à Saúde no Brasil: revisão da literatura. Ciência e Saúde Coletiva, 2016; 21(5): 1499-1509.

2. BEZERRA ACV. Discutindo o território e a territorialização na saúde: uma contribuição às ações de Vigilância em Saúde Ambiental. Revista de Geografia (UFPE), 2015; 32(3): 222-244.

3. BRASIL. Decreto no 5.209, de 17 de setembro de 2004. Regulamenta a Lei $n^{\circ} \mathbf{1 0 . 8 3 6}$, de 9 de janeiro de 2004, que cria o Programa Bolsa Família e dá outras providências. Brasília - DF, 2004.

4. BRASIL. Ministério da Cidadania. Secretaria Especial do Desenvolvimento Social. Guia para Acompanhamento das Condicionalidades do Programa Bolsa Família. Brasília - DF, 2020.

5. BRASIL. Ministério da Saúde. Portaria no 2.436, de 21 de setembro de 2017. Aprova a Política Nacional de Atenção Básica, estabelecendo a revisão de diretrizes para a organização da Atenção Básica, no âmbito do Sistema Único de Saúde (SUS). Diário Oficial da União, Brasília - DF, 2017.

6. CALVO MCM, et al. Estratificação de municípios brasileiros para avaliação de desempenho em saúde. Epidemiologia e Serviço de Saúde, 2016; 25(4): 767-776. 
7. CARVALHO AT, et al. Condicionalidades em saúde do programa Bolsa Família - Brasil: uma análise a partir de profissionais da saúde. Saúde e Sociedade, 2014; 23(4): 1370-1382.

8. CARVALHO DS, NASCIMENTO EPL, organizadoras. Dimensionamento da força de trabalho para a saúde do município de Fortaleza-CE. [s.n.], 2020; 170p.

9. FARIA RM. A territorialização da atenção primária à saúde no Sistema Único de Saúde e a construção de uma perspectiva de adequação dos serviços aos perfis do território. Revista Brasileira de Geografia Médica e da Saúde, Hygeia, 2013; 9(16): 131-147.

10. GOMES DR, et al. Desigualdades em saúde e a estratificação social no acesso aos serviços de saúde. Revista das Ciências da Saúde do Oeste Baiano, Hígia, 2016; 1(2): 19-33.

11. IBGE. 2016. Síntese de indicadores sociais: uma análise das condições de vida da população brasileira: 2016/IBGE, Coordenação de População e Indicadores Sociais. IBGE, 2016.

12. INSTITUTO BRASILEIRO DE INFORMAÇÃO EM CIÊNCIA E TECNOLOGIA (IBICT). Relatório de Progresso do Projeto Dimensionamento da Força de Trabalho em Secretarias de Saúde para a tomada de decisão em Gestão do Trabalho em Saúde a partir da coleta, estruturação e análise de dados quantitativos e qualitativos. Brasília-DF, 2020.

13. MACHADO CR, DAL POZ MR. Sistematização do conhecimento sobre as metodologias empregadas para o dimensionamento da força de trabalho em saúde. Saúde em Debate, 2015; 39 (104): 239-254.

14. MARQUES, AMP. Dimensionamento de recursos humanos na Atenção Básica - A experiência da Secretaria Municipal de Campinas (SP). In: MARQUES AMP, GOULART FAA, CASTRO JL, (Org.). Gestão do trabalho em saúde: experiências selecionadas do prêmio Inovação. Estudos e Análise 1. Brasília-DF: OPAS/OMS, 2016; 300p.

15. MONCLAR CN, et al. Dimensionamento da força de trabalho na atenção básica em Anápolis e Cachoeiro de Itapemirim: a parceria com o Ministério da Saúde do Brasil. In: CASTRO JL, VILAR RLA, COSTA TPT, (Org.). Trabalho e educação na saúde: análises e vivências. Natal: Una, 2020; 370p.

16. NASCIMENTO EPL, CARMONA SAMLD. A experiência da elaboração do dimensionamento na Atenção Básica da Secretaria Municipal de Saúde de Campinas In: FERLA AA, POSSA LB, GOSCH CS, TREPTE RF, (Org.). Dimensionamento da força de trabalho em saúde: gestão em ato e territórios em diálogo. $1^{a}$ ed. Porto Alegre: Rede Unida, 2020.No prelo.

17. NASCIMENTO EPL, et al. Dimensionamento de recursos humanos para o Sistema Único de Saúde de Campinas: Atenção Básica. In: Prêmio InovaSUS 2012/2013: valorização de boas práticas e inovação na gestão de trabalho na saúde. Ministério da Saúde. Secretaria de Gestão do Trabalho e da Educação na Saúde. Brasília, DF: Ministério da Saúde, 2015; 238p.

18. NASCIMENTO EPL, et al. Planejamento e dimensionamento da força de trabalho dos estabelecimentos da atenção primária à saúde. In: NASCIMENTO EPL, CARVALHO DS, CARMONA SAMLD, BARTHMANN VMC, (Org.). Planejamento e dimensionamento da força de trabalho em saúde: material didático para secretarias de saúde. $1^{\underline{a} e}$ ed. Brasília-DF: IBICT, 2020; 1: 49-84.

19. OLIVEIRA LCC, REIS AAC. Apontamentos sobre os desafios (ainda) atuais da atenção básica à saúde. Cadernos Saúde Pública, 2018; 34(8):1-14.

20. SANTOS M. A natureza do espaço: técnica, razão e emoção. 4ª̣ed. São Paulo: Editora da Universidade de São Paulo, 2006; 259p.

21. SANTOS M. Metamorfoses do espaço habitado. São Paulo: Hucitec, 1988; 28p.

22. VIANNA CMM, et al. Modelos econométricos de estimativa da força de trabalho: uma revisão integrativa da literatura. Physis: Revista de Saúde Coletiva, 2013; 23(3): 925-950. 